\title{
EXPLORING THE RECONFIGURABILITY OPTIONS OF MULTI-CARRIER CDMA IN COGNITIVE RADIO SYSTEMS
}

\author{
Sarath, D., Nolan, K.E.,Sutton, P.D., Doyle L.E. \\ Centre for Telecommunications Value-Chain Research (CTVR) \\ Trinity College Dublin \\ Rep. of Ireland \\ Email: sarathd@tcd.ie, keithnolan@mee.tcd.ie, suttonpd@tcd.ie, ledoyle@tcd.ie
}

\begin{abstract}
The ever-increasing demands for access to frequency spectrum and more efficient methods of using this spectrum involving multi-carrier techniques are driving the development of reconfigurable and cognitive wireless devices. The potential for, and the need to accommodate the potentially time-varying entropy in these wireless systems presents several design and implementation challenges. However, significant opportunities for innovation extending from the wireless terminal implementation to the business and market models also exist. This paper focuses on the physical (PHY) and medium-access control (MAC) layer issues of these emerging systems, highlighting the following three main contributions:
\end{abstract}

1. An introduction to reconfigurable multi-carrier code division multiple access (MC-CDMA)

2. An exploration of the use of reconfigurable MC-CDMA and its impact on the design of the physical (PHY) and medium access control (MAC) layers in a cognitive radio context.

3. An overview of the practical design and development considerations of this novel system which is being implemented on a real cognitive radio development testbed.

\section{INTRODUCTION}

Multi-carrier spectrum access techniques including orthogonal frequency division multiplex (OFDM) and multi-carrier code division multiple access (MC-CDMA) offer a number of significant advantages over single-carrier systems in volatile wireless channel environments. For example, OFDM is inherently more resilient to the effects of inter-symbol-interference (ISI) and multi-path fading [1]. In addition, OFDM is more spectrally-efficient due to the closely-packed carriers and may be equalised in the frequency domain, resulting in a less complex process when compared to time-domain equalisation techniques.

MC-CDMA has emerged as another feasible option for forward-looking multi-carrier communications systems $[2,3$, $4,5]$ by exploiting the flexibility and potential offered by the combination of OFDM and CDMA. The advantages offered by spreading the data over a wider bandwidth than the original signal has been utilized in CDMA implementations including the Interim Standard 95 (IS-95) and the Universal Mobile Telecommunications System (UMTS) 3G cellular communication systems. The ability to dynamically reconfigure these sys- tems according to the particular time, frequency, location and user objectives as a cognitive radio system helps to pave the way for new and innovative methods of wireless communication.

Cognitive radio (CR) can be described as a node in a network with the abilities to form an awareness of its environment and context, make decisions and inferences from this information combined with knowledge of the user's objectives, act in a manner that attempts to accomplish the user's objectives, and finally learn from these experiences for possible use in the future [6]. This cognitive functionality may not just be limited to the PHY and MAC layers but can extend its influence over all or many more of the layers in a communications stack. As a result of this cognitive functionality, the potential for spontaneous change and a relatively high level of system entropy exists as the cognitive radio adapts and preemptively acts to counteract and/or exploit change in the internal and external environment, and network topology.

This paper is organized as follows. Section II deals with a brief description of the MC-CDMA transceivers and the adopted implementation. Section III deals with the reconfigurability options, briefs on the potential of selective subcarrierMC-CDMA (SS-MC-CDMA) and the implementation on a cognitive radio development platform developed by CTVR. Section IV concludes with the scope for future research.

\section{MULTI-CARRIER CDMA AND IMPLEMENTATION}

\section{A. Multi-carrier CDMA (MC-CDMA)}

Two main variations of the multi-carrier spread spectrum systems are the MC-CDMA (frequency domain spreading) and MC direct sequence CDMA (MC-DS-CDMA) (time domain spreading). One way of looking at MC-CDMA is as a combination of CDMA and OFDM, resulting in better frequency diversity and higher data rates. In MC-CDMA, each symbol is spread using code chips and transmitted on several subcarriers. There is no necessity for the number of carriers to be equal to the code length; thus offering a degree of flexibility in our design. MC-DS-CDMA differs in the fact that the data is spread in time domain rather than in frequency; with each subchannel representing a regular DS-CDMA system. The basic principle outlining these is illustrated in Fig. 1. It illustrates the case of a single-user scenario where the data is spread using a code of length 4 and number of subcarriers is the same.

An extensive study of MC-DS-CDMA and its comparison with MC-CDMA can be found in $[4,5]$. The eminent advantage of MC-CDMA is the increase in bandwidth efficiency; the 


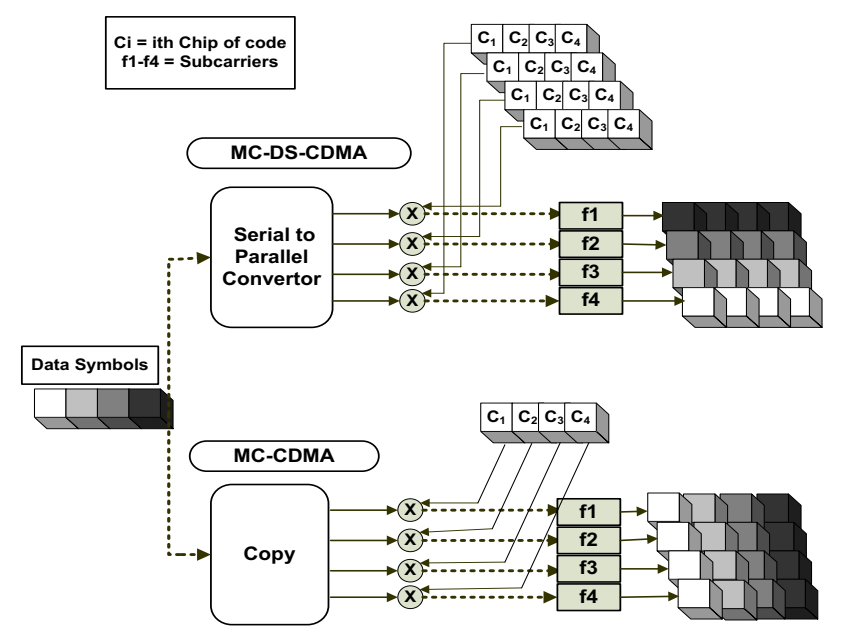

Figure 1: MC-CDMA and MC-DS-CDMA Transmitter principle

reason being the multiple access made possible through proper systems design using orthogonal codes. The additional flexibility offered by the possibility to use variable length codes offers greater resilience to error. It is also possible to interleave the data in both frequency [13] and time to exploit both time and frequency diversity and employ time-frequency (TF) spreading [4].

MC-CDMA and OFDM also have issues of high peak to average power ratios (PAPR), the challenges of synchronization in both the time and frequency domains and dealing with carrier frequency offset and multiple access interference (MAI) $[1,4,5,10]$.

\section{B. MC-CDMA Implementation}

The M-Modification of MC-CDMA [1] has been adopted for the initial implementation in our system. Consider the code of user $k$ having length $n$ as shown; where $c_{n}$ represents the chips of the individual code.

$$
C^{k}=\left\{c_{1}^{k} c_{2}^{k} c_{3}^{k} c_{4}^{k} \ldots \ldots \ldots \ldots \ldots c_{n}^{k}\right\}
$$

If there are $K$ users transmitting simultaneously using $n$ length orthogonal codes on $N_{\mathrm{fft}}$ number of subcarriers, the system can be represented as shown in Fig. 2. If there are $M$ data symbols before spreading, assuming all the subcarriers are filled by spread data, the condition is that

$$
N_{\mathrm{fft}}=M \times n .
$$

The transmitter side operation, as demonstrated in Fig. 2, consists of the addition of a cyclic prefix (CP) to mitigate the effects of intersymbol interference (ISI), the OFDM frame formation and parallel to serial conversion. The CP length is chosen such that it is greater than the delay spread of the channel so that the effects of multi-path are mitigated effectively. The hardware front end follows where digital to analog conversion (DAC) takes place and the signal is upconverted and transmitted on the required frequency channel. At the receiver side, the

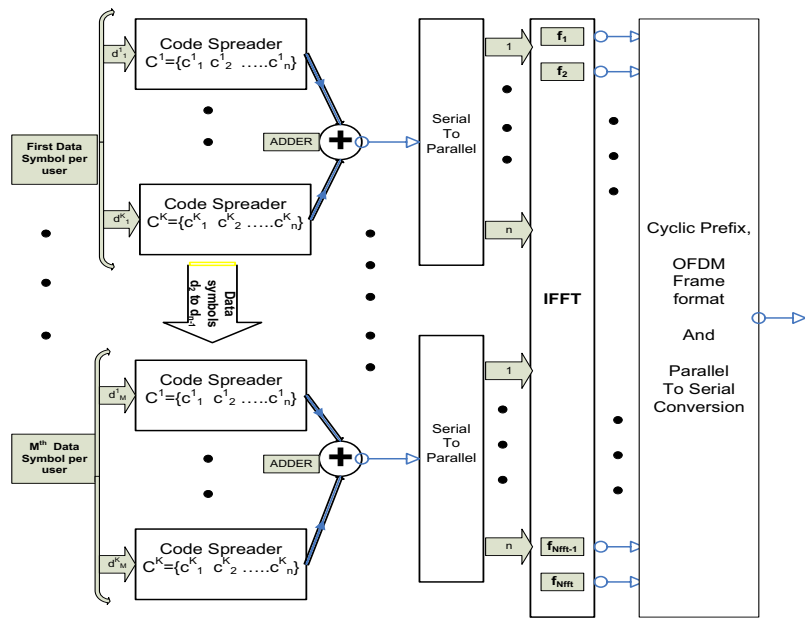

Figure 2: Implemented M-Modification of MC-CDMA

reverse operation takes place with $\mathrm{ADC}$, serial to parallel conversion, removal of $\mathrm{CP}$, extraction of symbols from the frame, code de-spreading and detection.

A limiting factor in order to restrict interference is that the number of users $K_{\max }$ be equal to the length of code $n$. Expanding on the basic MC-CDMA equation in $[11,16]$, we could form an equation for the M-modification as shown in Fig. 3. Here, $S(t)$ denotes the complex envelope of an M-ary PSK modulated MC-CDMA signal and $b_{i}$ denotes the M-ary PSK modulated data symbol from the stream $r$. The $u^{t h}$ chip of the $n$-length code of the $k^{\text {th }}$ user is denoted by $C_{r, u}^{k}$ and the symbol period is $T$ and $t \leq T$.

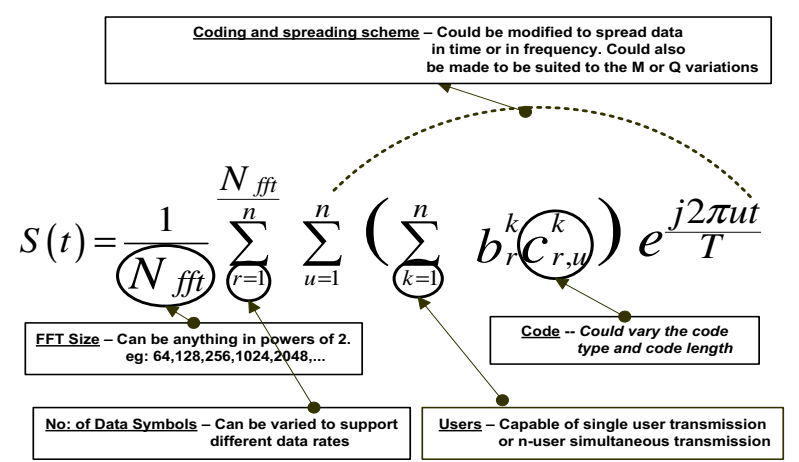

Figure 3: Reconfigurability in MC-CDMA

\section{RECONFIGURABILITY OPTIONS IN MC-CDMA}

Our software radio approach to employing MC-CDMA offers various levels of reconfigurability, while allowing dynamic adaptations with the capabilities of a Cognitive Radio. Fig. 3 illustrates the scope of reconfiguration. For convenience, these levels of reconfiguration are addressed as the primary degrees of freedom $\left(1^{\circ} \mathrm{DF}\right)$ and secondary degrees of freedom $\left(2^{\circ} \mathrm{DF}\right)$. $1^{\circ} \mathrm{DF}$ involves the parameters that are reconfigured almost in- 
stantly as reactions to triggers; such as the code type and length, FFT size, modulation method, channel coding and forward error correction (FEC). $2^{\circ} \mathrm{DF}$ involves the configurations that would lead to adaptation of the system as a whole. Once the $2^{\circ} \mathrm{DF}$ parameters are configured, default parameters for $1^{\circ} \mathrm{DF}$ is set or adjusted automatically to those specified in the trigger.

The advantage with this model is that the number of levels associated with each $1^{\circ} \mathrm{DF}$ and $2^{\circ} \mathrm{DF}$ can be varied and at a later stage, each of these manifests itself as one another. For example, when we consider a larger system, each block such as OFDM or MC-CDMA would serve $1^{\circ} \mathrm{DF}$ whereas the whole system configuration such as WLAN, emergency networks and ad-hoc networks would be the $2^{\circ} \mathrm{DF}$.

\section{A. Flexibility in Choice of Methods and Parameter Selection}

\section{Code Choice and MC-CDMA or MC-DS-CDMA}

The choice of code greatly depends on the scenario and is selected based on its orthogonality, correlation properties, implementation complexity and PAPR $[4,12]$. Walsh-Hadamard codes are simple to generate recursively and are particularly suited in a synchronous environment (eg: downlink in cellular networks) while in the case of asynchronous transmissions (eg: uplink in cellular networks), Gold codes have good correlation properties [1]. A CR approach offers the flexibility to employ a MC-CDMA system (using Walsh codes) in a downlink scenario and MC-DS-CDMA (using Gold codes) in the asynchronous uplink. Upon receiving the instruction from the cognitive engine, the change is effected by changing the direction of spreading in the coding block and reconfiguring its $1^{\circ} \mathrm{DF}$; thus offering not only the flexibility to change the length and type of code but also to change the system as such with minimal overhead and latency.

\section{Synchronization, Estimation and Detection}

$\mathrm{CR}$ allows the cognitive engine to dynamically choose the complexity of the pilots and synchronization preambles. If the cognitive engine indicates a hostile non-stationary channel, the pilots are spread in different subcarriers or 2D interleaved pilots would be distributed over several OFDM symbols [17].

Equalization at the receiver side is necessary to counter the channel effects leading to the loss of orthogonality between the signals. Single-user detection (SUD) using linear equalizers such as maximum ratio or equal gain combining, minimum mean square error or zero forcing; and multi-user detectors (MUD) using block equalizers are employed to achieve this $[5,4,1]$. When system parameters are known, techniques such as parallel or successive interference cancellation [1] can be utilized. Consider the case when the intelligent sensing mechanism informs the cognitive engine regarding the channel map. Based on its learning process, it can predict the channel and employ pre-equalization techniques at the transmitter; thus offering a choice of complexity depending on the scenario.

\section{Interleaving}

In the case when there are nulls or deep fades in the channel, information may be lost. Under these circumstances, the frequency diversity offered by MC-CDMA is exploited to the maximum by interleaving the data before mapping it onto the subcarriers. 2-D interleaving increases the diversity relative to time and frequency but at the expense of additional complexity and buffering, which makes it unsuitable for time-sensitive data.

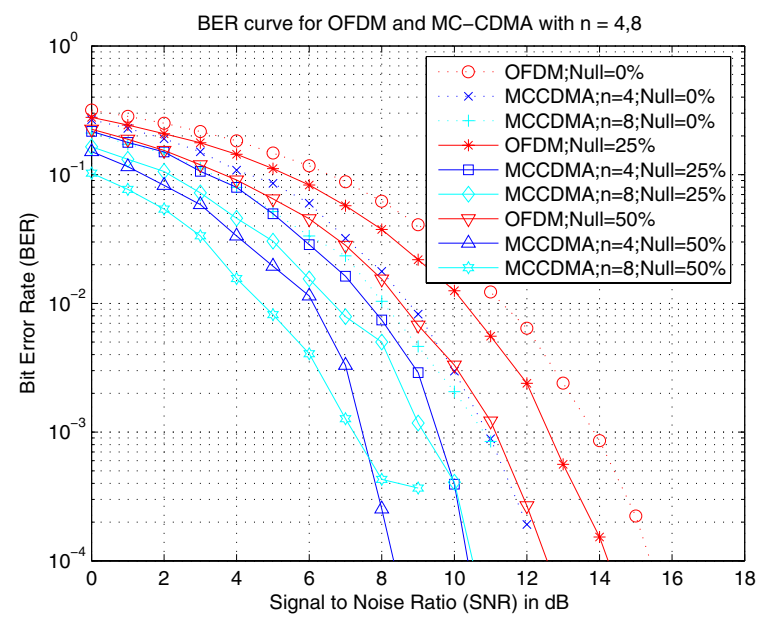

Figure 4: Effect of varying code length and subcarrier-nulling

\section{Null subcarriers (SS MC-CDMA - Selective Subcarrier MC- CDMA)}

Nolan K. [8] suggested the option of dynamic selection of subcarriers in an OFDM system using a channel mask and an enhanced training symbol (ETS). More recently, [16] has explored the option of nulling certain subcarriers (NC-OFDM) to offer spectrum agility and greater robustness to error. They had compared it to a similar MC-CDMA system and concluded that there is a data loss associated with subcarrier nullifying in MC-CDMA. The reason attributed to this was that the data in subcarriers are not independent as a result of the spreading operation. We would like to mention that in our system, IRIS, the data allocation to bins take place only after the subcarriers are selected. It is also worth noting that the authors [16] assumed the classical MC-CDMA system with data spread on all subcarriers where as in an M-Modification, the length of spreading code need not be equal to the number of subcarriers and thus it offers a lower dependency on the adjacent data. In our system model, since the data symbol is spread over several subcarriers, hostile channel performance at one sub band would still allow the recovery of the data symbol using the other subcarriers. Under a similar situation in OFDM, the data symbol would be corrupted by noise.

We have simulated a single user scenario to test the performance of the SS-MC-CDMA system in additive white Gaussian noise (AWGN) channel and the results are illustrated in Fig. 4. Graph is plotted for the same number of data symbols in each case with $N_{\mathrm{fft}}=256$. The data symbols are quadrature phase shift keying (QPSK) modulated with $\mathrm{CP}=2 \%$ of total symbol length. It has to be noted that in the case of a single 


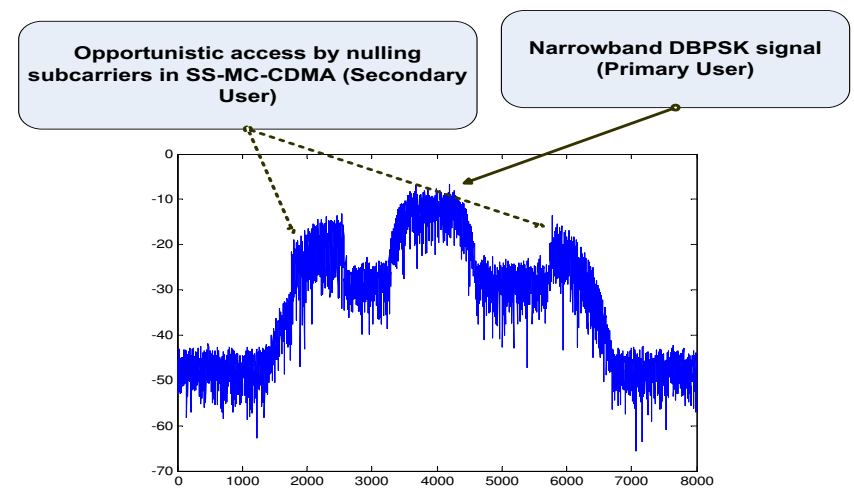

Figure 5: Real Instance of Spectrum Sharing using MC-CDMA

user, though the error resilience is high, the data rate reduces by a factor of the code length. Future work will involve the extensive analysis of the SS-MC-CDMA under different criteria and its potential in dynamic spectrum access (DSA). Fig. 5 illustrates the scenario of opportunistic access using an MC-CDMA signal. A differential binary phase shift keying (DBPSK) transmitter serves as the primary user (carrier frequency $=2.410$ $\mathrm{GHz}$, Data rate $=250 \mathrm{ksamples} / \mathrm{sec}$ ) whereas a SS-MC-CDMA transmitter ( 256 bin fft, 52 data subcarriers, $2 \mathrm{MHz}$ bandwidth) is the secondary user. The number of data subcarriers is chosen low to aid the visualisation of coexistance of signals.

\section{B. Design Considerations in the MAC Layer}

An ideal MAC layer for ad-hoc networks should be dynamic and adaptive in nature; should be distributed and capable of supporting variable data rates and should address the hidden node and exposed node problems. In an MC-CDMA based $\mathrm{CR}$, it should also be able to dynamically allocate codes and channels for simultaneous users with minimum collisions and should also account for the near-far problem by dynamic power control. Each frequency channel can be subdivided into smaller channels and different users could be allocated employing orthogonal codes. Different multichannel protocols have been studied, such as in $[18,19]$, but not many have analyzed the cross-layer optimization achievable by the PHY-MAC interactions. The design can be challenging but the flexibility offered by multi-carrier CDMA offers another dimension (i.e. code) for allocation as shown in Fig. 6. On employing MC-CDMA, the allocation criteria on each frequency channel breaks down further into determining the solution of a function of the frequency, available subcarriers $f_{\text {subcarr }}$ ), time, space and code, which could be further exploited in a CR scenario to minimize collisions and maximize throughput.

$$
\text { Allocate }=\text { fn }\left(\text { freq, } f_{\text {subcarr }}, \text { time }, \text { space }, \text { code }\right)
$$

\section{Implementation in IRIS}

Initially designed by Mackenzie P. [7], the IRIS (Implementing Radio in Software) platform was developed to facilitate the research and to test CRs. The current implementation of the IRIS includes enhancements by Nolan K. and Sutton P. $[8,9]$

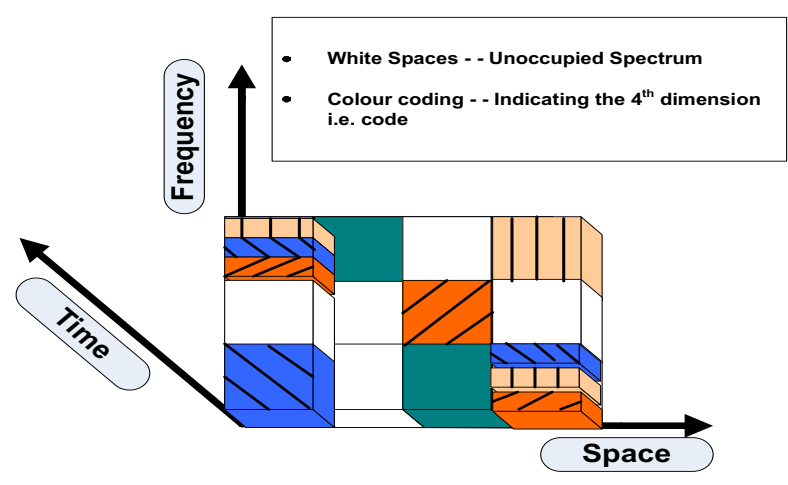

Figure 6: 4D representation of the MC-DS-CDMA system allocation example

and accommodates varying levels of abstraction and support for cross-layer optimizations.

The modules are realized in $\mathrm{C}++$ and are stored as dynamic link libraries (dll) for quick access. Modules can be anything from signal adders and multipliers to OFDM, MCCDMA modems. The blackboard enables to observe desired parameters in the process chain, thus playing a vital role in the cognitive cycle. Further details of the IRIS platform can be found in $[7,8,9]$.

Example of an adaptation cycle using IRIS: At this point, we assume the availability of a system parameter identified as the channel map (CM). Fig. 7 illustrates a conceptual reconfiguration process explaining the reconfiguration using three blocks; namely coding, channel map and IFFT. Initially a DS-CDMA system, upon receiving a trigger as a read-out from the channel map, the system's decision block decides to switch to a multi-carrier system and an IFFT block is added to provide the multi-carrier capabilities. The $1^{\circ} \mathrm{DF}$ parameters are set to default or to the values specified by the decision block. At a later instance $t_{n}$, if the system decides that only one user needs to be supported on a fairly good channel, it can avoid the coding and decoding complexity and overhead. Setting the code length to one, it defaults to an OFDM system. The triggers specifying these changes can be incorporated into the cognitive capabilities of the radio. It thus would act on its previous knowledge of the channel and system or it would intelligently sense the spectrum and switch accordingly. Note that the inclusion of new blocks not only offers additional $1^{\circ} \mathrm{DF}$ but also allows modification of the inherited $1^{\circ} \mathrm{DF}$.

\section{CONCLUSION AND FUtURE WORK}

We have analyzed the various reconfigurability options in a MC-CDMA system and have illustrated the flexibility options offered by their variants in a cognitive radio. We also highlight the scope for SS-MC-CDMA, which would not only counter the issues pertaining to deep channel fades, but also open the possibilities of spectrum sharing or opportunistic access. In a system with such high degree of reconfigurability, dynamic adaptations and spontaneous changes would result in potentially high level of entropy. Future work would involve the 


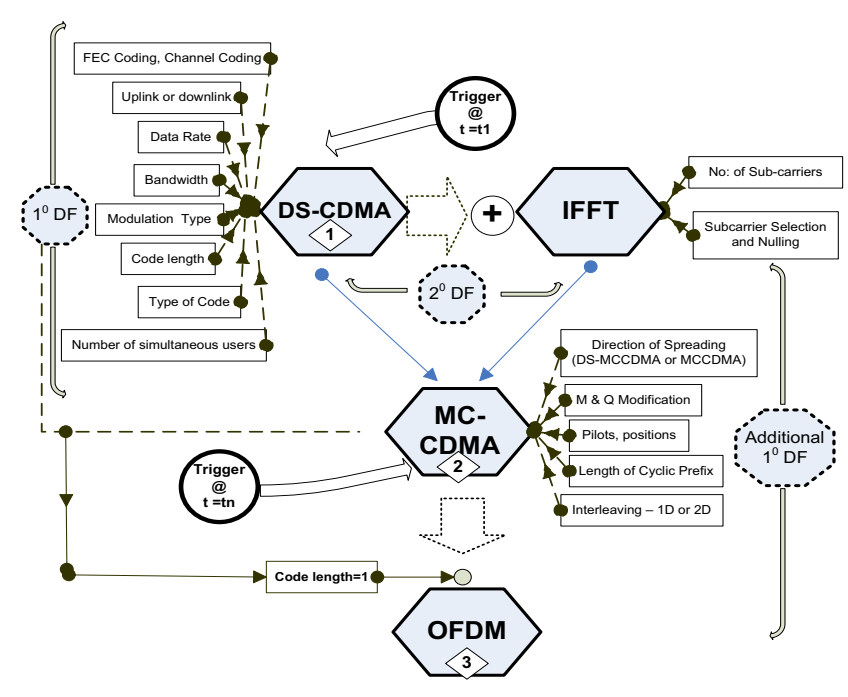

Figure 7: Example of an Adaptation Cycle

development of the channel map (CM) function and would be focussed on establishing parameters derived analytically or empirically by means of real measurements. These would help identify the triggers to switch from one configuration to another in CR. The development of a MAC protocol that supports these functionalities of a PHY layer is also targeted, with the implementation and testing based on the IRIS reconfigurable platform.

\section{ACKNOWLEDGMENTS}

This work is based upon work supported by Science Foundation Ireland under Grant No: 03/CE3/1405 as part of the Centre for Telecommunications Value Chain Research(CTVR), at Trinity College Dublin, Ireland.

\section{REFERENCES}

[1] Fazel K. and Kaiser S, Multi-Carrier and Spread Spectrum Systems, Wiley, England, 2003.

[2] Yee. N, Linnartz. J and Fettweis. G, Multi-carrier CDMA for indoor wireless radio networks, Proc. International Symposium on Personal, Indoor and Mobile Radio Communications (PIMRC'93), Yokohoma, Japan, Pages: 109-113,Sept 1993.

[3] Hara S. and Prasad R. Overview of Multi-carrier CDMA, IEEE Transactions on Communications, Pages: 195-198, Volume: 47, Issue: 2, Feb 1999.

[4] Hanzo et al, Single- and Multi-Carrier DS-CDMA: Multi-User Detection, Space-Time Spreading, Synchronisation and Standards, IEEE Press and Wiley, England, 2003.

[5] Hanzo et al, OFDM and MC-CDMA: for Broadband Multi-User Communications, WLANs and Broadcasting, IEEE Press and Wiley, England, 2003.

[6] Haykin S., Cognitive Radio: Brain-Empowered Wireless Communications, IEEE Journal on Selected Areas in Communications, Feb 2005.
[7] Mackenzie P. Reconfigurable Software Radio Systems, PhD Thesis, Trinity College Dublin, Ireland, 2004.

[8] Nolan K.E. Reconfigurable OFDM Systems, PhD Thesis Trinity College Dublin, Ireland, 2005.

[9] Sutton P., Doyle L. and Nolan K.E., A Reconfigurable Platform for Cognitive Networks, Proc. 1st International Conference on Cognitive Radio Oriented Wireless Networks and Communications (CROWNCOM'06), Jun 2006.

[10] Yee N. and Linnartz J.P, Controlled Equalization of MultiCarrier CDMA in an Indoor Rician Fading Channel, Proc. 44th Vehicular Technology Conference (VTC'94), Volume:3, Pages: 1665-1669, Jun 1994

[11] Choi B-J, Kuan E-L and Hanzo L, Crest-factor Study of MCCDMA and OFDM, Proc. 50th Vehicular Technology Conference (VTC'99), Volume: 1, Pages: 233-237, Netherlands, Sept 1999

[12] Kang K, Choi K and Shin S-K, Reduced Search for Optimum Code Sets to Reduce PAPR in MC-CDMA Systems, Proc. 5th International Symposium on Wireless Personal Multimedia Communications, Volume:1, Pages: 135-139, Oct 2002

[13] Furudate M, Ishikawa $\mathrm{H}$ and Suzuki T, Evaluation of MCCDMA with Frequency Interleaving Technique in Frequency Selective Fading Channels, Special Section on Multi-Carrier Signal Processing Techniques for Next Gen Mobile Communications, The Institute of Electronics, Information and Communication Engineers (IEICE), Volume: E88-B, No. 2, Feb 2005

[14] Weiss T.A and Jondral F.K., Spectrum Pooling: An Innovative Strategy for the Enhancement of Spectrum Efficiency, IEEE Communications Magazine, Volume: 42, Issue: 3, Pages: 8 14, Mar 2004

[15] Jondral F.K, Software-Defined Radio - Basics and Evolution to Cognitive Radio, EURASIP Journal on Wireless Communications and Networking, Volume: 2005, Issue: 3, Pages: 275283, Apr 2005

[16] Rajbanshi et al., Comparative Study of Frequency Agile Data Transmission Schemes for Cognitive Radio Transceivers, 2nd Annual International Wireless Internet Conference - International Workshop on Technology and Policy for Accessing Spectrum (TAPAS'06), Boston, USA, Aug 2006.

[17] Kaiser S and Hoeher P, Performance of Multi-Carrier CDMA Systems with Channel Estimation in Two Dimensions, Proc. 8th IEEE International Symposium on Personal, Indoor and Mobile Radio Communications (PIMRC'97), Volume: 1, Pages: 115119, Sept 1997.

[18] Orfanos G et al., An Adaptive MAC Protocol for MC-CDMA Adhoc Wireless LAN, Proc. 16th IEEE International Symposium on Personal, Indoor and Mobile Radio Communications (PIMRC'05), Volume: 4, Pages: 2734-2741, Sept 2005.

[19] Scorza G.B, Sacchi C. and Granelli F., An Adaptive MAC-PHY Approach for Medium Access Control in VBR MC-CDMA Systems, Proc. 1st International Conference on Multimedia Services Access Networks (MSAN'05), Pages: 96-100, June 2005. 